\section{Effect of patient characteristics on medication adherence among patients with type 2 diabetes mellitus: a cross-sectional survey}

By: Abdullah, NF (Abdullah, Nor Fadhilah) $)^{[1,2]}$; Khuan, L (Khuan, Lee ${ }^{[1]}$; Theng, CA (Theng, Cheong Ai) ${ }^{[3]}$; Sowtali, SN (Sowtali, Siti Noorkhairina) ${ }^{[4]}$; Juni, MH (Juni, Muhamad Hanafiah) ${ }^{[5]}$

View Web of Science ResearcherID and ORCID

\section{CONTEMPORARY NURSE}

Volume: 55 Issue: 1 Pages: 27-37

DOI: $10.1080 / 10376178.2019 .1583067$

Published: JAN 22019

Document Type: Article

View Journal Impact

\section{Abstract}

Background: The prevalence of diabetes mellitus (DM) is steadily increasing worldwide, with a significant DM population in Asian countries. Adherence to medications is important to achieve good glycaemic control among patients with DM. Thus, patients' adherence to their medication regimen should be determined to optimise DM management. Aims: To determine medication adherence and the relationship between patient profile and medication adherence among patients with type 2 DM (T2DM). Design: Cross-sectional survey. Methods: This study was conducted in a public hospital in Selangor, Malaysia, from December 2016 to June 2017. Data was obtained through administration of the Medication Compliance Questionnaire and an electronic medical records database. Multivariate logistic regression analysis was used to determine the predictors of medication adherence. Results: A total of 232 (95.9\% response rate) patients participated in this study. The overall percentage of medication adherence among patients with DM was $55.2 \%$. The majority of participants were female (53.4\%), Malay (47.0\%), aged 41-64 years (55.2\%; mean age, 56.69 years), married ( $84.5 \%)$, unemployed (60.8\%) and attended secondary school (53.9\%). The factors independently associated with adherence were ethnicity (odds ratio [OR], 1.43; $95 \%$ confidence interval [Cl]: 1.03-1.99) and haemoglobin Alc (HbAlc) level (OR, 2.71; 95\% Cl: 1.56-4.72). Conclusions: The medication adherence among patients with DM in a public hospital in Selangor, Malaysia was low. A health intervention emphasising patient-centred care is warranted to improve DM patients' adherence to prescribed medication. Considering that Malaysia has a multi-ethnic population, the patients' ethnicity and their HbAlc levels need to be considered in the implementation of any intervention to improve medication adherence. Impact statement: Medication adherence is influenced by individual patients' characteristics. To improve adherence to the medication regimen, nurses should consider patients' profiles.

\section{Keywords}

Author Keywords: adherence; diabetes; medication; patient characteristics; nursing

KeyWords Plus: GLYCEMIC CONTROL; HEALTH CLINICS; CARE

\section{Author Information}

Reprint Address: Khuan, L (reprint author)

+ Univ Putra Malaysia, Fac Med \& Hlth Sci, Dept Nursing \& Rehabil, Serdang 43400, Selangor, Malaysia. Addresses:

+ [1] Univ Putra Malaysia, Fac Med \& HIth Sci, Dept Nursing \& Rehabil, Serdang 43400, Selangor, Malaysia

+ [2] Univ Sultan Zainal Abidin, Fac Med, Dept Nursing, Gong Badak 21300, Terengganu, Malaysia

+ [3] Univ Putra Malaysia, Fac Med \& Hlth Sci, Dept Family Med, Serdang 43400, Selangor, Malaysia

+ [4] Int Islamic Univ Malaysia, Dept Profess Nursing Studies, Kulliyyah Nursing, Jalan Hosp Campus, Kuantan 25100, Pahang, Malaysia

+ [5] Univ Putra Malaysia, Fac Med \& Hlth Sci, Dept Community Hlth, Serdang 43400, Selangor, Malaysia

E-mail Addresses: leekhuan@upm.edu.my

Funding

\begin{tabular}{|l|l|}
\hline Funding Agency & Grant Number \\
\hline Universiti Putra Malaysia, Selangor, Malaysia under the FRGS grant & 5524771 \\
\hline
\end{tabular}

View funding text

Publisher

ROUTLEDGE JOURNALS, TAYLOR \& FRANCIS LTD, 2-4 PARK SQUARE, MILTON PARK, ABINGDON OX14 4RN, OXON, ENGLAND

\section{Journal Information}

Impact Factor: Journal Citation Reports

Categories / Classification

Research Areas: Nursing

Web of Science Categories: Nursing

\section{Citation Network}

In Web of Science Core Collection

0

Times Cited

Create Citation Alert

\section{5}

Cited References

View Related Records

\section{Use in Web of Science}

Web of Science Usage Count

2

Last 180 Days Since 2013

Learn more

This record is from:

Web of Science Core Collection

- Science Citation Index Expanded

- Social Sciences Citation Index

Suggest a correction

If you would like to improve the quality of the data in this record, please suggest a correction. 


\section{Cited References: 35}

1. Treatment non-adherence among patients with poorly controlled type 2 diabetes in ambulatory care settings in southwestern Nigeria

By: Adisa, Rasaq; Fakeye, Titilayo 0.

AFRICAN HEALTH SCIENCES Volume: 14 Issue: 1 Pages: 1-10 Published: 2014

2. Factors associated with good glycemic control among patients with type 2 diabetes mellitus

Times Cited: 27

By: Ahmad, Nur Sufiza; Islahudin, Farida; Paraidathathu, Thomas

JOURNAL OF DIABETES INVESTIGATION Volume: 5 Issue: 5 Pages: 563-569 Published: SEP 2014

3. Medication adherence in patients with type 2 diabetes mellitus treated at primary health clinics in Malaysia

Times Cited: 62

By: Ahmad, Nur Sufiza; Ramli, Azuana; Islahudin, Farida; et al.

PATIENT PREFERENCE AND ADHERENCE Volume: 7 Pages: 525-530 Published: 2013

4. Title: [not available]

Times Cited: 1

By: Akmal Naziah, A.

Factors influencing glycaemic control in older people with type 2 DM on oral hypoglycemic therapy and attending a primary health care centre in Negeri Sembilan,

Malaysia Published: 2011

Publisher: Universiti Putra Malaysia, Malaysia

5. Standards of Medical Care in Diabetes-2013

Times Cited: 2,240

Group Author(s): Amer Diabet Assoc

DIABETES CARE Volume: 36 Supplement: 1 Pages: S11-S66 Published: JAN 2013

6. Standards of medical care in diabetes-2019

Times Cited: 2

Group Author(s): American Diabetes Association

Diabetes Care Volume: 42 Supplement:Suppl Pages: 1 Published: 2019

7. Strategies to enhance patient adherence: Making it simple

Times Cited: 1

By: Atreja, A.; Bellam, N.; Levy, S. R.

Medscape Health Network Volume: 7 Issue: 1 Pages: 1-18 Published: 2005

8. Medication adherence in type 2 diabetes patients: study of patients in Alimosho General Hospital, Igando, Lagos, Nigeria

Times Cited: 20

By: Awodele, Olufunsho; Osuolale, Jemeela A.

AFRICAN HEALTH SCIENCES Volume: 15 Issue: 2 Pages: 513-522 Published: 2015

9. Adherence to oral anti-diabetic drugs among patients attending a Ghanaian teaching hospital.

Times Cited: 10

By: Bruce, Suliasnaia P; Acheampong, Franklin; Kretchy, Irene

Pharmacy practice Volume: 13 Issue: 1 Pages: 533 Published: 2015 Jan-Mar (Epub 2015 Mar 15)

10. Determinants of medication adherence among adults with type 2 diabetes mellitus in three Malaysian public health clinics: a cross-sectional study By: Chew, Boon-How; Hassan, Noor-Hasliza; Sherina, Mohd-Sidik

PATIENT PREFERENCE AND ADHERENCE Volume: 9 Pages: 639-648 Published: 2015

11. Influence of Chinese cultural health beliefs among Malaysian Chinese in a suburban population: a survey

Times Cited: 9

By: Chew, K. S.; Tan, T. W.; Ooi, Y. T.

SINGAPORE MEDICAL JOURNAL Volume: 52 Issue: 4 Pages: 252-256 Published: APR 2011

12. Medication adherence and achievement of glycaemic targets in ambulatory type 2 diabetic patients.

By: Chua, S. S.; Chan, S. P.

Journal of Applied Pharmaceutical Science Volume: 1 Issue: 4 Pages: 55-59 Published: 2011

13. Peripheral arterial disease in people with diabetes

Times Cited: 1,038

By: Clark, N; Sheehan, P; Edmonds, M; et al.

Group Author(s): Amer Diabet Assoc

DIABETES CARE Volume: 26 Issue: 12 Pages: 3333-3341 Published: DEC 2003

14. Factors Contributing To Non-Adherence To Medication among Type 2 Diabetes Mellitus In Patients Attending Tertiary Care Hospital In South India

By: Divya, S.; Nadig, P.

Asian J. Pharm. Clin. Res Volume: 8 Pages: 2 Published: 2015

15. The association between the measurement of adherence to anti-diabetes medicine and the $\mathrm{HbAlc}$

By: Doggrell, Sheila A.; Warot, Servane 
16. Physical activity and type 2 diabetes. Recommandations of the SFD (Francophone Diabetes Society) diabetes and physical activity working group

By: Duclos, M.; Oppert, J. -M.; Verges, B.; et al.

Group Author(s): SFD Diabetes Phys Act Working Grp

DIABETES \& METABOLISM Volume: 39 Issue: 3 Pages: 205-216 Published: MAY 2013

17. Factors Affecting Medica-tion Non Adherence in Type 2 Sudanese Diabetic Patients

Times Cited: 4

By: El - Hadiyah, TM; Madani, AM; Abdelrahim, HM; et al.

Pharmacology \& Pharmacy Volume: 7 Pages: 141-146 Published: 2016

[Show additional data]

18. Adherence to Therapies in Patients with Type 2 Diabetes

Times Cited: 261

By: Garcia-Perez, Luis-Emilio; Alvarez, Maria; Dilla, Tatiana; et al.

DIABETES THERAPY Volume: 4 Issue: 2 Pages: 175-194 Published: DEC 2013

19. Predictors of Diabetes Self-Management among Type 2 Diabetes Patients

Times Cited: 6

By: Gunggu, Azylina; Thon, Chang Ching; Lian, Cheah Whye

JOURNAL OF DIABETES RESEARCH Article Number: 9158943 Published: 2016

20. Title: [not available]

Times Cited: 1

By: GUPTA AK

ENVIRON ENER ENGN S Pages: 1 Published: 2000

21. Title: [not available]

Times Cited: 6

Group Author(s): Institute for Public Health, Ministry of Health, Malaysia

National Health and Morbidity Survey 2015: Report on Smoking Status Among Malaysian Adults Published: 2015 accessed 10 October 2018

Publisher: The Ministry, Kuala Lumpur

22. Comparing diabetic patient characteristics related to stated medication adherence in a rural vs. urban community in Korea

Times Cited: 3

By: Kim, H.; Kim, H. -S.; Bowman, J. D.; et al.

JOURNAL OF CLINICAL PHARMACY AND THERAPEUTICS Volume: 41 Issue: 1 Pages: 40-46 Published: FEB 2016

23. DETERMINING SAMPLE SIZE FOR RESEARCH ACTIVITIES

Times Cited: 2,027

By: KREJCIE, RV; MORGAN, DW

EDUCATIONAL AND PSYCHOLOGICAL MEASUREMENT Volume: 30 Issue: 3 Pages: 607-\& Published: 1970

24. Self-care, Self-efficacy, and Glycemic Control of Koreans With Diabetes Mellitus

Times Cited: 7

By: Lee, Haejung; Ahn, Sukhee; Kim, Yongsuk

ASIAN NURSING RESEARCH Volume: 3 Issue: 3 Pages: 139-146 Published: SEP 2009

25. Health-related quality of life in patients with diabetic foot problems in Malaysia.

Times Cited: 15

By: Mazlina, M; Shamsul, A S; Jeffery, FA Saini

The Medical journal of Malaysia Volume: 66 Issue: 3 Pages: 234-8 Published: 2011-Aug

26. Title: [not available]

Times Cited: 4

Group Author(s): Ministry of Health; Malaysia

Clinical practice guidelines. management of type 2 diabetes mellitus Published: 2015 accessed May 17, 2018

27. Predictive Validity of a Medication Adherence Measure in an Outpatient Setting

Times Cited: 1,292

By: Morisky, Donald E.; Ang, Alfonso; Krousel-Wood, Marie; et al.

JOURNAL OF CLINICAL HYPERTENSION Volume: 10 Issue: 5 Pages: 348-354 Published: MAY 2008

28. Diabetes knowledge and adherence among geriatric patient with type 2 diabetes mellitus

Times Cited: 9

By: Omar, Marhanis Salihah; San, Kong Lai.

Int J Pharm Pharm Sci Volume: 6 Issue: 3 Pages: 103-6 Published: 2014

29. Dietary behaviors among patients with Type 2 diabetes mellitus in Yogyakarta, Indonesia

Times Cited: 4

By: Primanda, Y; Kritpracha, C; Thaniwattananon, P.

Nurse Media J Nurs Volume: 1 Pages: 211-23 Published: 2011

30. Trends in prevalence of diabetes in Asian countries

Times Cited: 203

By: Ramachandran, Ambady; Snehalatha, Chamukuttan; Shetty, Ananth Samith; et al.

WORLD JOURNAL OF DIABETES Volume: 3 Issue: 6 Pages: 110-117 Published: JUN 152012

Showing 30 of $35 \quad$ View All in Cited References page 
